\title{
A influência do gênero e idade no desempenho das habilidades locomotoras de crianças de primeira infância
}

CDD. 20.ed. 152.3

796.1
Dayana da Silva OLIVEIRA*

Ilana Santos de OLIVEIRA*

Maria Teresa CATTUZZO*
*Escola Superior de Educação Física, Universidade de Pernambuco.

\section{Resumo}

Na primeira infância é esperada uma melhora no desempenho de habilidades locomotoras com o avanço da idade. Pode também haver diferenças entre meninos e meninas devido à especificidade da tarefa motora. Este estudo teve como objetivo analisar o desempenho de crianças em habilidades locomotoras de acordo com idade e gênero. 0 desempenho locomotor de 389 pré-escolares de três a cinco anos da cidade do Recife - PE foi avaliado mediante o TGMD-2. Os resultados mostraram que: crianças mais velhas apresentaram melhor desempenho motor; no geral, meninos obtiveram melhor desempenho no correr, salto horizontal, deslize lateral e escore total locomotor, e meninas no saltitar com um pé aos cinco anos de idade; na análise da progressão do desenvolvimento motor, comportamentos motores mais complexos ocorreram após aqueles considerados menos complexos. Conclui-se que o desenvolvimento locomotor dos pré-escolares recifenses obedeceu aos principios da progressividade e do aumento da complexidade, entretanto, meninos e meninas mostraram diferenças no seu desempenho dependendo da tarefa.

Palavras-chave: Desempenho psicomotor; Pré-escolares; Desenvolvimento infantil; Destreza motora.

\section{Introdução}

É durante a primeira infância, período que corresponde à etapa da pré-escola e início da escola primária, que ocorrem as mudanças e adaptaçóes mais expressivas no sistema motor humano ${ }^{1-2}$. A aquisição de habilidades locomotoras (ex.: correr, saltar), de controle de objetos (ex.: lançar e receber uma bola) e estabilizadoras (ex.: equilíbrio em um só pé), parecem ser fundamentais para todo o desenvolvimento motor posterior, uma vez que formarão a base para ações relacionadas ao esporte, dança e jogos ${ }^{1,3-5}$.

Assim, o domínio dessas habilidades motoras é uma das mais importantes tarefas do início da infância $^{6}$, pois crianças que não desenvolvem de maneira eficiente seus padrôes de movimento terão dificuldades de adquirir níveis aceitáveis de desenvolvimento em açōes motoras futuras ${ }^{7-8}$. Segundo CLARK $^{9}$ a finalidade de um ótimo desenvolvimento motor é alcançar um "status" de "indivíduo competente", ou seja, aquele que sabe realizar as ações, motiva-se para realizá-las, mostra confiança no seu desempenho e continua movimentando-se por toda sua vida.
Pensando o comportamento motor infantil como um sistema aberto, dinâmico e complexo e o desenvolvimento motor como um processo multicausal expresso pelo aumento de complexidade nas ações motoras $^{10-11}$, é preciso considerar os fatores relativos ao organismo, ao ambiente e à especificidade da tarefa. Por exemplo, o desempenho das habilidades motoras deve melhorar com o avanço da idade, uma vez que o desenvolvimento motor está sujeito ao processo maturacional, mecanismo biológico relacionado à idade cronológica que leva à melhoria de todas as funções orgânicas ${ }^{5,12}$. Mas, estudiosos do desenvolvimento motor são enfáticos ao afirmar que o domínio nas habilidades motoras também depende da oportunidade e qualidade da prática nessas tarefas ${ }^{1,5,13-16}$. Além disso, a especificidade da tarefa também deve ser considerada para um melhor entendimento de como ocorre o processo de desenvolvimento motor, pois as restrições inerentes a cada tarefa podem afetar o seu desempenho.

Focalizando especificamente as habilidades locomotoras pode-se observar que elas abrangem desde 
as primeiras tentativas da caminhada independente até açōes mais complexas como saltar e correr em várias formas e direçōes ${ }^{17} \mathrm{e}$ sabe-se que, mesmo na primeira infância, é possível verificar diferenças entre gêneros para essas habilidades. MaLINA et al. ${ }^{6}$ apontam que o maior tamanho do corpo e comprimento de alavancas podem fazer com que os meninos mostrem melhor desempenho motor grosso, especialmente em tarefas que exijam deslocamento do corpo no espaço. Além disso, condições impostas culturalmente (como advertências ou incentivos), que parecem ser oferecidas diferentemente dependendo do gênero da criança, são fatores que podem influenciar o desempenho dessas habilidades.

Embora alguns estudos tenham mostrado a similaridade entre o desempenho de meninos e meninas ${ }^{18-22}$, outros apontam diferenças entre os gêneros, mostrando melhor desempenho de meninos ${ }^{23-24}$ ou meninas ${ }^{25-26}$. Essa diferença entre os gêneros também parece estar associada ao tipo de tarefa locomotora, por exemplo, os meninos parecem ter especial domínio no

\section{Método}

Este é um estudo transversal, descritivo e de enfoque quantitativo $^{32}$. Está baseado em um levantamento epidemiológico de abrangência municipal, intitulado ELOS-PRÉ (Estudo Longitudinal de Observação da Saúde e Bem-estar da Criança em Idade Pré-escolar), que foi aprovado pelo Comitê de Ética local (CAAE - 0096.0.097.000-10).

A população alvo foi constituída por crianças em idade entre três e cinco anos matriculadas em escolas da rede pública e privada de educação na área de abrangência da Gerência Regional de Educação do Recife Norte e correr $^{24,27}$ e as meninas, por sua vez, teríam na habilidade saltitar ${ }^{28}$. Adicionalmente, deve-se levar em consideração que as habilidades locomotoras apresentam diferenças em termos de sua complexidade efetora, e isso parece afetar o momento do seu surgimento. Por exemplo, é esperado que após a criança ter estabelecido firmemente o padrão da corrida, desenvolva-se a habilidade de galopar, um padrão locomotor assimétrico que combina locomoção e saltos, apresentando uma maior complexidade de movimentos ${ }^{29}$. Isso ressalta a importância da análise do aumento da complexidade efetora, que é esperado ocorrer no processo de desenvolvimento motor $^{12,30-31}$, e que não foi enfatizado nos estudos revisados sobre o tema.

Considerando que o desenvolvimento motor infantil deve progredir e aumentar em complexidade, e que este é um processo influenciado por fatores do indivíduo, do ambiente e da tarefa, pode-se questionar se meninos e meninas se diferem quanto à qualidade das ações no desempenho em habilidades locomotoras, e se as diferenças estão ligadas à especificidade da tarefa.

TABELA 1 - Total de crianças, distribuídas de acordo com gênero e idade (anos), participantes da amostra do teste de habilidades locomotoras na cidade do Recife - PE.

DP - desvio padrão da idade em anos;

Méd - média da idade em anos.

\begin{tabular}{lccccccc}
\hline & \multicolumn{2}{c}{3 anos } & \multicolumn{2}{c}{$\mathbf{4}$ anos } & \multicolumn{2}{c}{$\mathbf{5}$ anos } & \multirow{2}{*}{ Total } \\
\cline { 2 - 6 } & $\mathbf{n}$ & $\mathbf{M e d}(\mathbf{D P})$ & $\mathbf{n}$ & $\mathbf{M e d}(\mathbf{D P})$ & $\mathbf{n}$ & $\mathbf{M e d}(\mathbf{D P})$ & \\
\hline Meninos & 29 & $3,45(0,30)$ & 70 & $4,17(0,32)$ & 115 & $5,46(0,29)$ & 214 \\
Meninas & 25 & $3,49(0,29)$ & 72 & $4,59(0,29)$ & 78 & $5,44(0,27)$ & 175 \\
Total & 54 & & 142 & & 193 & & 389 \\
\hline
\end{tabular}

A coleta de dados foi realizada no período letivo das escolas, durante o segundo semestre de 2010. Para a verificação do desempenho motor das crianças
Recife Sul, e foi realizada a seleção dos sujeitos adotando a amostragem por conglomerados em múltiplos estágios. $\mathrm{O}$ banco de dados para as medidas de desempenho motor foi de 555 crianças. $\mathrm{Na}$ amostra da presente investigação o critério adotado para a inclusão dos sujeitos foi a criança ter completado as duas tentativas de cada uma das seis habilidades locomotoras filmadas. Foram excluídas as crianças cujos dados de filmagem apresentassem baixa qualidade para análise. $\mathrm{O}$ tamanho amostral final do presente estudo foi de 389 crianças, sendo 214 meninos e 175 meninas (TABELA 1). 
de objetos em crianças de três a 10 anos de idade, recentemente validado para crianças brasileiras ${ }^{33}$. $\mathrm{Na}$ aplicação do teste, um avaliador treinado explicava e demonstrava cada uma das habilidades e, em seguida, a criança a executava três vezes. A primeira execução realizada pela criança era um ensaio. Se o avaliador identificasse qualquer problema de entendimento por parte da criança, ele realizava uma nova demonstração, e na sequência, as duas tentativas da criança eram coletadas.

As ações motoras foram filmadas por Câmera Digital Sony Cyber-Shot DSC-H20 (10.1 Megapixels) e posteriormente pontuadas segundo critérios da lista de checagem estabelecida pelo teste ${ }^{7}$. Foi utilizado o reprodutor de vídeo Media Player Classic ("free download"), em velocidade lenta para análise dos vídeos. A decodificação dos dados de filmagem foi feita por dois especialistas. A Concordância Entre Observadores $(\mathrm{CEO})^{32}$ do somatório de cada tentativa foi de $86 \%$, e cada critério pontuado diferentemente entre os avaliadores foi revisto em conjunto e concordado entre eles.

As variáveis independentes foram: gênero (meninos, meninas), idade (três, quatro e cinco anos). A variável

\section{Resultados}

Os resultados dos desempenhos em habilidades locomotoras são mostrados a seguir. A ANOVA "twoway" ( 3 idades $x 2$ gêneros) mostrou que houve efeito de idade $(\mathrm{F}=20,25 ; \mathrm{p} \leq 0,001$; Poder observado $=$ $1,00)$, de gênero $(F=3,36 ; p=0,002$; Poder observado $=0,961)$ e efeito de interação idade $\mathrm{x}$ gênero $(\mathrm{F}$ = 2,71; $\mathrm{p}=0,009$; Poder observado = 0,906).

Análises posteriores mostraram que as crianças mais velhas sempre foram melhores que as crianças mais novas em todas as habilidades e no escore total locomotor. Crianças de quatro anos mostraram melhor desempenho que as crianças de três anos nas habilidades: saltitar com um pé ( $\mathrm{p}=0,000 ; \mathrm{ES}=0,89)$, saltar o obstáculo ( $\mathrm{p}=0,001$; ES $=0,51)$ e escore total locomotor $(\mathrm{p}=0,000 ; \mathrm{ES}=0,85)$. Crianças de cinco anos mostraram melhor desempenho que as de três anos nas seis habilidades locomotoras [correr $(p=0,000 ; E S=0,61)$, galopar $(p=0,000$; $\mathrm{ES}=0,68)$, saltitar $(\mathrm{p}=0,000 ; \mathrm{ES}=1,31)$, saltar o obstáculo $(\mathrm{p}=0,002 ; \mathrm{ES}=0,50)$; salto horizontal ( $\mathrm{p}$ $=0,000 ; \mathrm{ES}=0,62)$, deslize lateral $(\mathrm{p}=0,000 ; \mathrm{ES}=$ dependente foi o desempenho em seis habilidades locomotoras (correr, galopar, saltitar com um pé, saltar o obstáculo, salto horizontal e deslize lateral), além do escore total locomotor. Para pontuação do desempenho: foi atribuído um ponto se o critério da lista de checagem fosse atendido ou zero, caso contrário. A medida de desempenho foi o somatório dos pontos das duas tentativas. $\mathrm{O}$ escore total locomotor foi o somatório dos pontos de todas as seis habilidades.

Os testes de Shapiro-Wilk (utilizado para a amostra das crianças de três anos de idade) e KolmogorovSmirnov (para os demais sujeitos) indicaram que os dados apresentaram normalidade, então foram adotados testes paramétricos. A descrição das medidas de tendência central e dispersão foram apresentadas com média e desvio padrão (DP), respectivamente. Para comparação entre idades e gêneros nas seis habilidades locomotoras e escore total locomotor, utilizou-se ANOVA "two-way" (3 idades x 2 gêneros) e, para os contrastes posteriores, foi utilizado o teste de Bonferroni. Para esses cálculos foi utilizado o pacote estatístico SPSS 10.0. O tamanho do efeito ("Effect Size" - ES) foi calculado por meio da média e desvio padrão, usando o "software” WinPepi versão 11.18 ("free download").

$0,99)$,] e no escore total locomotor ( $\mathrm{p}=0,000$; ES = 1,58). As crianças de cinco anos mostraram melhor desempenho que as de quatro anos nas habilidades galopar $(p=0,006 ; E S=0,35)$, saltitar com um pé $(p=0,000 ; E S=0,44)$, salto horizontal $(p=0,000$; $\mathrm{ES}=0,42)$, deslize lateral $(\mathrm{p}=0,000 ; \mathrm{ES}=0,61) \mathrm{e}$ no escore total locomotor ( $\mathrm{p}=0,000 ; \mathrm{ES}=0,75)$.

Relativo ao efeito de gênero, meninos foram melhores que as meninas nas habilidades correr ( $p$ $=0,001 ; \mathrm{ES}=0,34)$, salto horizontal $(\mathrm{p}=0,032$; $\mathrm{ES}=0,26)$, deslize lateral $(\mathrm{p}=0,016 ; \mathrm{ES}=0,22)$ e no escore total locomotor ( $\mathrm{p}=0,027$; $\mathrm{ES}=0,15$ ).

Relativo ao efeito de interação idade x gênero, houve interação para a habilidade saltitar com um pé $(\mathrm{p}=0,003$; Poder observado $=0,873)$ e para o escore total locomotor ( $\mathrm{p}=0,015$; Poder observado $=0,743)$. Aos cinco anos de idade, as meninas foram melhores que os meninos no saltitar com um pé $(p<0,001 ; E S=0,54)$. Relativo ao escore total locomotor, meninos foram melhores que meninas aos três anos de idade ( $\mathrm{p}=0,027$; $\mathrm{ES}=0,62)$. 
* diferença $(p<0,05)$ entre crianças de $3 \mathrm{e}$ 4 anos;

$\dagger$ diferença $(p<0,05)$ entre crianças de $3 \mathrm{e}$ 5 anos;

$\S$ diferença $(p<0,05)$ entre crianças de $4 \mathrm{e}$ 5 anos.

* diferenças estatisticamente significativas $(p$ $<0,05$ ).

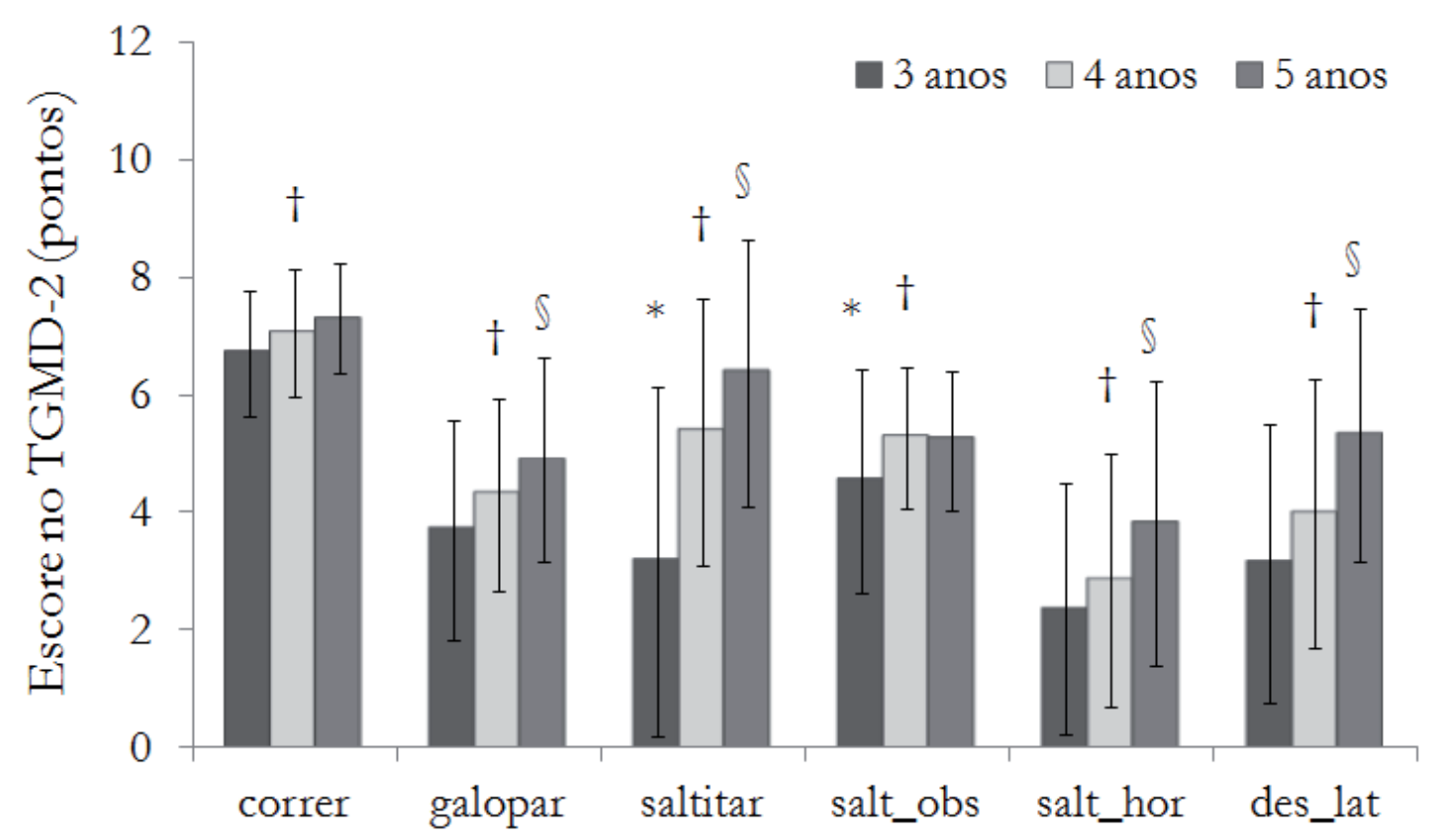

FIGURA 1 - Média e desvio padrão dos escores no TGMD-2 das crianças de três, quatro e cinco anos nas habilidades correr, galopar, saltitar, saltar o obstáculo (salt_obs), salto horizontal (salt_hor) e deslize lateral (desl_lat). Recife - PE.

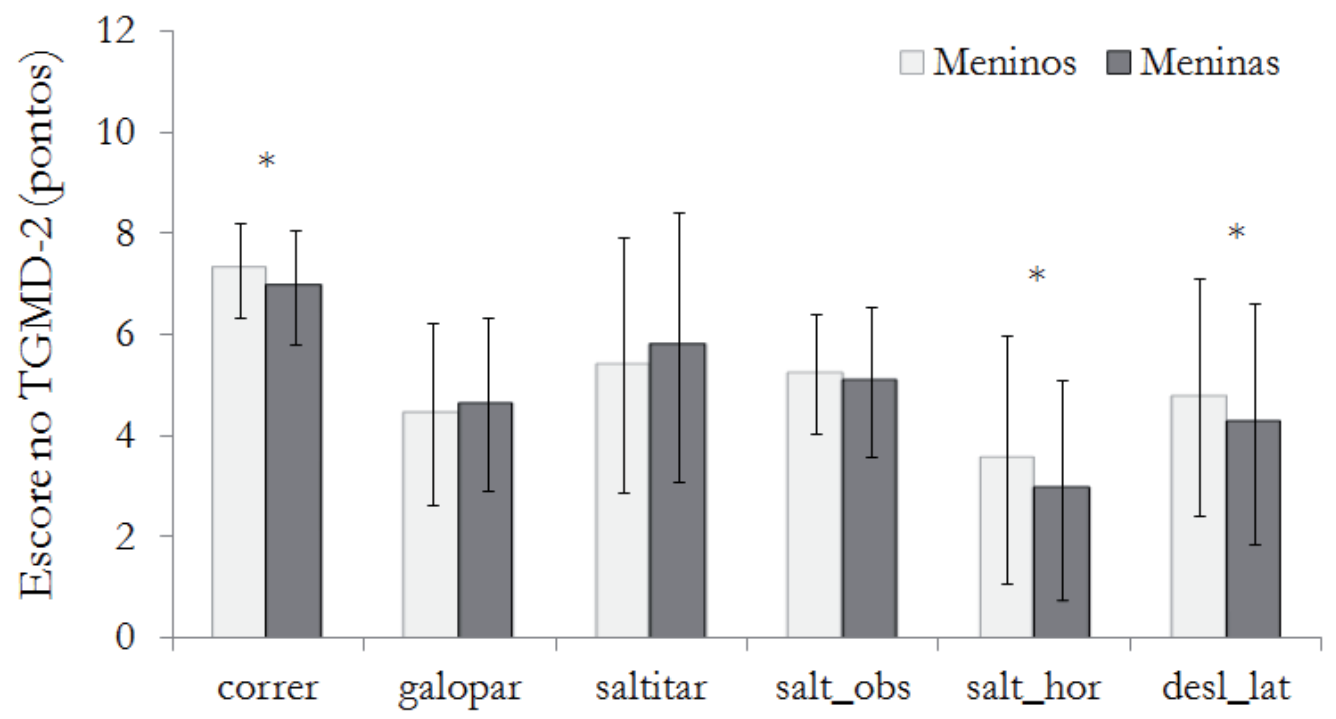

FIGURA 2 - Média e desvio padrão dos escores no TGMD-2 de meninos e meninas de três a cinco anos nas habilidades correr, galopar, saltitar, saltar o obstáculo (salt_obs), salto horizontal (salt_hor) e deslize lateral (desl_lat). Recife - PE.

A FIGURA 3 resume as diferenças significativas no desempenho das habilidades locomotoras entre as crianças dessa amostra, considerando o intervalo de tempo médio de um ano entre as idades estudadas. Essa figura é um meio de expressar o aumento da complexidade efetora no desenvolvimento das habilidades locomotoras realtivo às três idades investigadas, revelando que: a) crianças de quatro anos, quando comparadas às de três anos, mostraram melhor desempenho em habilidades que exigem combinação de corrida e saltos (saltitar e saltar o obstáculo); b) crianças de cinco anos, quando comparadas às de quatro anos, são melhores em habilidades que exigem a combinação de locomoção e saltos e controle coordenativo (galopar, saltitar, salto horizontal e deslize lateral). 

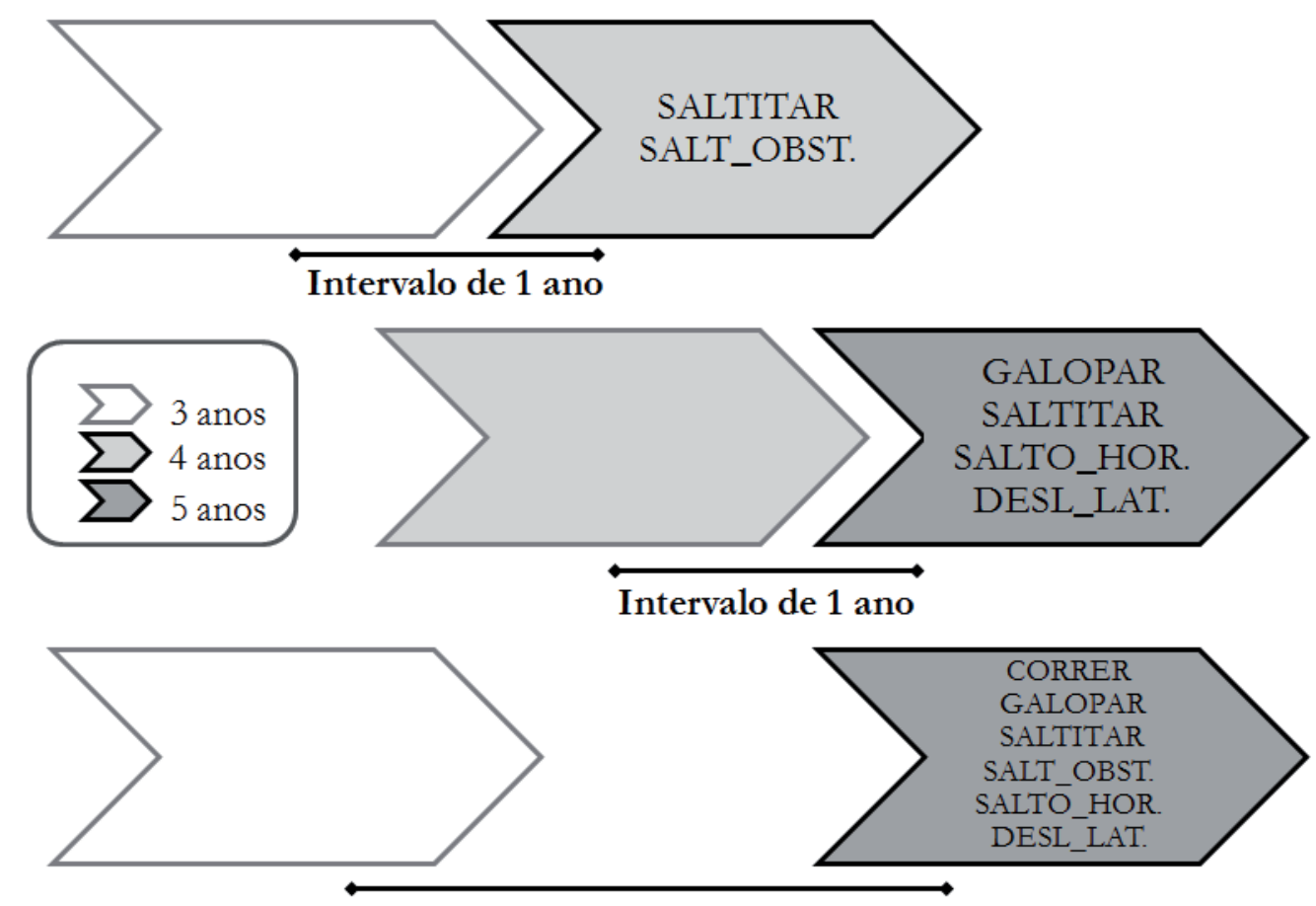

Intervalo de 1 ano

Intervalo de 2 anos

FIGURA 3 - Resumo das melhoras significativas encontradas entre as idades no desempenho nas habilidades locomotoras correr, galopar, saltitar, saltar o obstáculo (SALT_OBS), salto horizontal (SALT_HOR), deslize lateral (DESL_LAT), de crianças de três a cinco anos da cidade do Recife - PE.

\section{Discussão}

O objetivo deste estudo foi analisar o desempenho de crianças de primeira infância em seis habilidades locomotoras, comparando-as de acordo com idade, gênero.

\section{Análise do desempenho de acordo com as idades}

Com relação à comparação entre idades (três, quatro e cinco anos) comprovou-se um melhor desempenho motor das crianças mais velhas, corroborando os achados de outros estudos, que também identificaram a melhora no comportamento motor de crianças de primeira infância com o avanço da idade cronológica ${ }^{18,23,27,34}$. Além disso, as crianças mais velhas deste estudo (cinco anos) foram as que obtiveram melhor desempenho em todas as habilidades locomotoras. Estes dados fornecem suporte para a noção de que o comportamento motor infantil pode ser explicado a partir de uma abordagem sistêmica ${ }^{11,35}$ na qual a diferenciação, um princípio sistêmico fundamental, determina que os sistemas abertos partem de um estado no qual seus desempenhos são pouco especializados e pouco funcionais e avançam, diferenciado-se, tornandose cada vez mais especializados. A especialização das várias partes que compõe um sistema viabiliza a sua própria evolução. Reforçando essa visão, de acordo com $\mathrm{TANI}^{31}$, o aumento da diversidade e da complexidade no desempenho de habilidades motoras são processos fundamentais, por meio dos quais as crianças avançam para os níveis superiores de complexidade em seu desenvolvimento motor.

\section{Análise do desempenho de acordo com gênero e especificidade da tarefa}

Um dos principais objetivos deste estudo foi verificar se meninos e meninas são diferentes no desempenho de habilidades locomotoras. As análises apontaram que no escore total locomotor os meninos apresentaram melhores desempenhos quando comparados às meninas. Esse achado é suportado por outros estudos com crianças de primeira infância ${ }^{23}$ e de segunda infância ${ }^{24}$.

Os resultados também revelaram que os meninos obtiveram melhor desempenho motor comparado às 
meninas, nas habilidades de correr, salto horizontal e deslize lateral. CARVALHAL e VASCONCELOS-RAPOSO ${ }^{24}$ também encontraram melhor desempenho dos meninos na corrida, e segundo GABBARD ${ }^{28}$ há uma tendência de maior proficiência no correr de meninos na primeira infância. Outros estudos corroboram nossos achados, revelando índices médios superiores para os meninos em comparação às meninas na habilidade de salto horizontal ${ }^{23}$, e melhores resultados para os meninos na habilidade de deslize lateral ${ }^{22}$. Um possível argumento é que pode haver maior oferta de práticas motoras vigorosas e incentivo dedicado aos meninos ${ }^{18}$. CARVALHAL e VASCONCELOS-RAPOSO ${ }^{24}$ em um estudo com crianças de sete e oito anos de idade afirmaram que meninos estão mais envolvidos com brinquedos que contêm componentes móveis e promovem uma maior atividade motora em espaços mais amplos (por exemplo: bolas e carros), inversamente às atividades geralmente vivenciadas pelas meninas, que envolvem brincadeiras mais estáticas e comportamentos mais verbais do que motores, como por exemplo, brincar com bonecas. Apesar de a presente investigação ser composta por crianças de primeira infância (três, quatro e cinco anos), é possível que as demandas culturais relativas às ofertas de brinquedos e brincadeiras sejam estabelecidas desde muito cedo na vida dos indivíduos.

Por outro lado, as meninas foram melhores na habilidade de saltitar em um pé aos cinco anos de idade, o que já foi visto em outros estudos ${ }^{22,25-26}$. $\mathrm{GABBARD}^{28}$ propôs que meninas são mais eficientes em habilidades locomotoras específicas que requerem controle motor fino, flexibilidade e equilíbrio, como o saltitar em um pé, confirmando os achados da presente investigação. Destaca-se, no entanto, que isso só ocorreu na idade mais avançada analisada neste estudo (cinco anos). Também estudando a diferença entre gêneros no desempenho motor, GAR$\mathrm{CIA}^{36}$ revelou que meninos e meninas diferiam em suas capacidades físicas e preferências de habilidades motoras em seu cotidiano: entre as meninas, pareceu haver um maior interesse pela interação social e cooperação, ao invés da manipulação de bolas ou pelo sucesso das atividades. Os meninos, por sua vez, esforçaram-se para destacar-se, agindo de maneira competitiva, individualizada e egocêntrica, inclusive em suas próprias relações de aprendizagem.

Afonso et al..$^{27}$, ao investigar crianças de três a 10 anos, evidenciaram diferenças entre gêneros na corrida e no saltar o obstáculo, sendo que meninos foram superiores nessas habilidades aos quatro, cinco e nove anos e as meninas obtiveram melhor desempenho na habilidade de saltar o obstáculo na idade de oito anos. Assim, tantos os dados da literatura e os achados do presente estudo permitem sugerir que no desenvolvimento motor infantil pode existir diferença de desempenho para ambos os gêneros, dependendo do tipo de habilidade e/ou a idade do indivíduo.

Em que pese o fato de que este estudo não teve por objetivo coletar dados sobre as peculiaridades do contexto de brincadeiras de meninos e meninas, pode-se sugerir que as diferenças encontradas entre gêneros são devido às distintas interaçôes entre os elementos que influenciam o comportamento motor: a) ambientais (reforço social diferenciado para os comportamentos motores em cada gênero); b) organísmicas (idade cronológica e/ou sexo) e c) da tarefa (diferentes demandas percepto-motoras). Se a relação entre esses elementos é não linear e dinâmica, isso implicaria que suas variações podem levar a diferentes resultados comportamentais ${ }^{10}$. Assim, poder-se-ia hipotetizar que, para os meninos, o maior tempo dedicado à prática de atividades mais vigorosas, somado ao maior incentivo social para tais ações motoras, pode ter afetado positivamente seu desenvolvimento locomotor grosso.

As habilidades de locomoção têm diferentes restriçōes que afetam a sincronia e a sequência na qual emergem $^{29}$. A sequência teoricamente esperada para o desenvolvimento locomotor inicia-se com o correr (estabelecido firmemente em torno dos dois anos de idade), seguida pelo galopar (o primeiro padrão locomotor assimétrico que demanda força e coordenaçãa, e que combina corrida e saltitos, aparecendo entre os dois e três anos). A seguir, ocorre o surgimento dos saltos (que dependem da capacidade do sistema postural e equilíbrio), emergindo entre os três e quatro anos e, dentre eles, o saltitar com um pé é aquele que apresenta maior dificuldade, pela grande exigência da capacidade de equilíbrio dinâmico; em seguida, deveria ocorrer o domínio do deslocamento lateral que envolve a combinação dos elementos passada e salto e o controle coordenativo, surgindo entre o correr e o saltitar ${ }^{5,29}$.

Considerando o comportamento motor infantil um sistema aberto e dinâmico, dois processos são fundamentais: aumento da diversidade e da complexidade do comportamento ${ }^{31}$. Entende-se por aumento de diversidade o aumento na quantidade de elementos do comportamento e por aumento da complexidade, o aumento na interação entre elementos do comportamento. Os achados deste estudo revelaram que: a) crianças de quatro anos, quando comparadas às de três, tiveram melhor desempenho em duas das seis habilidades analisadas (saltitar em um pé e saltar o obstáculo); b) crianças de cinco anos apresentaram 
melhores desempenhos que as de quatro anos, em um maior número de habilidades (quatro habilidades) e principalmente em habilidades de maior complexidade (galopar, saltitar, salto horizontal e deslize lateral). O salto horizontal, particularmente, pareceu ser a habilidade em que as crianças mais novas (três e quatro anos) demonstraram especial dificuldade no seu desempenho. A habilidade salto horizontal exige uma sofisticada interação entre os momentos de tomada de impulso, fase de voo e aterrissagem, tornando-a, portanto, bastante complexa e de grande dificuldade em sua realização; c) ao comparar crianças de cinco e três anos as diferenças ficam ainda mais evidentes, pois as crianças mais velhas apresentaram melhores desempenhos em todas as seis habilidades locomotoras e no escore locomotor total, apresentando um tamanho do efeito que variou de médio a alto. Esse dado de magnitude do efeito reforça a importância da mudança de desempenho entre as idades. Assim, pode-se sugerir que os resultados do presente estudo corroboram a proposição de $\mathrm{TANI}^{31}$ sobre o aumento na diversidade e complexidade no comportamento motor.

Ainda que o presente estudo não tenha objetivado verificar os determinantes didáticos-pedagógicos que interferem no desempenho em habilidades motoras, é oportuno destacar que a falta de prática sistematizada pode não fornecer suporte necessário para atingir níveis de desempenho esperados para cada faixa etária. Alguns fatores como o tempo insuficiente ofertado às vivências motoras para obtenção de mudanças desenvolvimentais ${ }^{37}$ e a falta de otimização das estratégias de aula, como instruçôes e demonstrações pouco compreensíveis e possível carência de diversidade da prática $^{21}$ servem como alerta para os profissionais da área, que por sua vez, devem buscar uma melhor compreensão do processo de desenvolvimento motor, subsidiando futuras propostas de práticas motoras. Vale ressaltar que o tipo de profissional responsável pelas práticas é um fator que influencia, pois um estudo recente de LEMOS et al. ${ }^{38}$ revela que a atuação do professor especialista com pré-escolares foi decisiva para promover melhoras no desenvolvimento motor grosso das crianças e conceder a elas, possibilidades para continuar a desenvolver-se de maneira adequada.

Finalmente, considerando o caráter descritivo do presente estudo, corrobora-se a afirmação de Hopkins et al. ${ }^{39}$ enfatizando que a relação entre descrição e explicação no comportamento motor deve reconhecer uma categoria intermediária, a qual eles denominaram de 'explicações descritivas': uma vez que esteja firmemente calcado em teoria, as descrições sobre "o que" e "como" são passos essenciais no fornecimento de alguma explicação científica, ressaltando a importância da presente investigação.

Pode-se concluir que as crianças mais velhas mostraram melhores desempenhos com relação às mais novas nas habilidades locomotoras; meninos apresentaram melhor desempenho locomotor no escore total e nas habilidades: correr, salto horizontal e deslize lateral; já as meninas apresentaram melhor desempenho na habilidade de saltitar com um pé. Neste estudo com crianças recifenses de primeira infância, o comportamento motor infantil mostrou desenvolver-se em termos do aumento da quantidade, ou seja, número de habilidades locomotoras, e da qualidade das habilidades locomotoras desenvolvidas, evidenciando o aumento da diversidade e complexidade no comportamento motor humano.

\begin{abstract}
The influence of gender and age on locomotor skill performance in children of early childhood

In early childhood the performance of locomotor skills is expected to be improved in with advancing age. Differences between boys and girls due to the specificity of motor task may exist. This study aimed to analyze the locomotor skills performance of children regarding age and gender. The locomotor performance of 389 preschool children from three to five years from the city of Recife - PE was evaluated by TGMD-2. The results showed that: older children outperformed the younger ones; in general, the boys outperformed the girls in the skills to run, horizontal jump, slide and in the locomotor total score, and the girls had a better performance in the skill to hop at five years; at the analysis of the progression of motor development, more complex motor behaviors occurred after those considered less complex. It is concluded that the locomotor development of preschoolers from Recife followed the principles of progressivity and complexity, however, boys and girls showed differences on the performance depending on the task.
\end{abstract}

KEY WORDS: Psychomotor performance; Preschool child; Infant development; Motor skills. 


\section{Referências}

1. Clark JE. On the problem of motor skill development. J Phys Educ Recreat Dance. 2007;78:1-58.

2. Castro MB. A influência do contexto nas habilidades motoras fundamentais de pré-escolares e escolares [dissertação]. Porto Alegre (RS): Universidade Federal do Rio Grande do Sul; 2008.

3. Tani G. Educação física na pré-escola e nas quatro primeiras séries do ensino de primeiro grau: uma abordagem de desenvolvimento I. Kinesis. 1987;3:19-41.

4. Manoel EJ. Desenvolvimento motor: implicações para a educação física escolar I. Rev Paul Educ Fís. 1994;8:82-97.

5. Gallahue DL, Ozmun JC. Compreendendo o desenvolvimento motor: bebês, crianças, adolescentes e adultos. 3a ed. São Paulo: Phorte; 2005.

6. Malina RM, Bouchard C, Bar-Or O. Growth, maturation, and physical activity. 2nd ed. Champaign: Human Kinetics; 2004.

7. Ulrich DA. Test of gross motor development-2. Austin: Prod-Ed; 2000.

8. Gallahue DL, Donnelly FC. Developmental physical education for all children. 4th ed. Champaign: Human Kinetics; 2003.

9. ClarkJE. Motor development: what is it and why does it matter in preventing obesity? IOM Committee on Obesity Prevention Policies for Young Children (2010). Testimony 6.7.10. [citado 5 nov. 2011]. Disponível em: http://www.docstoc.com/docs/91301087/Jane-Clark.

10. Smith LB, Thelen E. Development as a dynamic system. Trends Cogn Sci. 2003;7:343-8.

11. Cattuzzo MT, Beltrão NB, Campos CMC, Guerra ERF. A multicausalidade desenvolvimental: uma abordagem sistêmica da proficiência motora e da prática da atividade física. In: Cattuzzo MT, Caminha IO, organizadoras. Fazer e pensar ciência em educação física: livro 1. João Pessoa: UFPB; 2012. cap. 3.

12. Haywood KM. Life span motor development. Champaign: Human Kinetics; 1986.

13. Garcia C, Garcia L, Floyd J, Lawson J. Improving public health through early childhood movement programs. J Phys Educ Recreat Dance. 2002;73:27.

14. Clark JE. From the begnning: a developmental perspective on movement and mobility. Quest. 2005;57:37-45.

15. Williams HG, Pfeiffer KA, O'Neill JR, et al. Motor skill performance and physical activity in preschool children. Obesity. 2008:16:1421-6.

16. Goodway JD. What's skill got to do with it? A developmental approach to promoting physical activity in Urban youths. J Phys Educ Recreat Dance. 2009;80:1-60.

17. Oliveira JA. Padrões motores fundamentais: implicações e aplicações na educação física infantil. Rev Interação. 2002;6.

18. Valentini NC. Percepçôes de competência e desenvolvimento motor de meninos e meninas: um estudo transversal. Movimento. 2002;8:51-62.

19. Goodway J, Grome H, Ward P. Effects of motor skill instruction on fundamental motor skill development. Adapt Phys Activ Q. 2003;20:298-314.

20. Hume C, Okely A, Bagley S, Telford A, Crawford D, Salmon J. Does weight status influence associations between children's fundamental movement skills and physical activity? Res Q Exerc Sport. 2008;79:158-65.

21. Brauner LM, Valentini NC. Análise do desempenho motor de crianças participantes de um programa de atividades físicas. Rev Educ Fís/UEM. 2009;20:205-16.

22. Xavier CTS. A escola e o desenvolvimento motor em escolares [dissertação]. Belém: Universidade Federal do Pará; 2009.

23. Paim MCC. Desenvolvimento motor de crianças pré-escolares entre 5 e 6 anos. Lect Educ Fís Desportes: Rev Digital. 2003;8. [citado 27 maio 2011]. Disponível em: http://www.efdeportes.com/efd58/5anos.htm.

24. Carvalhal M, Vasconcelos-Rapouso J. Diferenças entre gêneros nas habilidades: correr, saltar, lançar e chutar. Motricidade. 2007;3:44-56.

25. Van Beurden E, Zask A, Barnett L, Dietrich U. Fundamental movement skills: how do primary school children perform? The Move it Groove it'program in rural Australia. J Sci Med Sport. 2002;5:244-52.

26. Hardy LL, King L, Farrell L, MacNiven R, Howlett S. Fundamental movement skills among Australian preschool children. J Sci Med Sport. 2010;13:503-8.

27. Afonso GH, Freitas DL, Carmo JM, et al. Desempenho motor: um estudo normativo e criterial em crianças da Região Autónoma da Madeira, Portugal. Rev Port Ciênc Desporto. 2009;9:160-74.

28. Gabbard C. Lifelong motor development. 4th ed. San Francisco: Pearson; 2004.

29. Haywood KM, Getchell, N. Desenvolvimento motor ao longo da vida. 5a ed. Porto Alegre: Artmed; 2010.

30. Clark JE, Whitall J. What is motor development? The lessons of the history. Quest. 1989;41:183-202.

31. Tani G. Liberdade e restrição do movimento no desenvolvimento motor da criança. In: Krebs RJ, Copetti F, Beltrame, TS. Discutindo o desenvolvimento infantil. Santa Maria: Pallotti; 1998. 
32. Thomas JR, Nelson JK. Métodos de pesquisa em atividade física. 3a ed. Porto Alegre: Artmed; 2002.

33. Valentini NC. Validity and reliability of the TGMD-2 for Brazilian children. J Mot Behav. 2012;44:275-80.

34. Silveira CRA, Gobbi LTB, Caetano MJD, Rossi ACS, Candido RP. Avaliação motora de pré-escolares: relações entre idade motora e idade cronológica. Lect Educ Fís Desportes: Rev Digital. 2005;82. [citado 27 maio 2011]. Disponível em: http://www.efdeportes.com/efd83/avalia.htm.

35. Bertalanffy L. von. Teoria geral dos sistemas. 2a ed. Petrópolis: Vozes; 1975.

36. Garcia C. Gender differences in young children's interactions when learning fundamental motor skills. Res Q Exerc Sport. 1994;65:213.

37. Pansera SM, De Paula PR, Valentini NC. Educação física no ensino infantil: sua influência no desempenho das habilidades motoras fundamentais. Cinergis. 2008;9:24-32.

38. Lemos AG, Avigo EL, Barela JA. Physical education in kindergarten promotes fundamental motor skill development. Adv Phys Educ. 2012;2:17-21.

39. Hopkins B, Kalverboer AF, Geuze RHM. Epilogue: description versus explanation. In: Kalverboer AF, Geuze RHM, editors. Motor development in early and later childhood: longitudinal approaches. Cambridge: Cambridge University Press, 1993.

ENDEREÇO

Maria Teresa Cattuzzo

Universidade de Pernambuco

Escola Superior de Educação Física

R. Arnóbio Marques, 310

13405-260 - Recife - SP - BRASIL

e-mail: mtcattuzzo@hotmail.com
Recebido para publicação: 09/03/2012

1a. Revisão: 18/01/2013

2a. Revisão: 19/06/2013

Aceito: 08/07/2013 